\title{
Hydrogel Spacer may Allow Prostate Re-irradiation even in Patients with Ulcerative Colitis: A Case Report
}

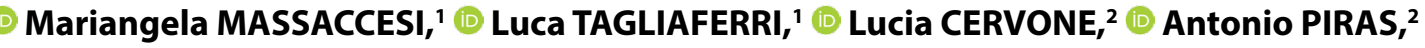 \\ Debastiano MENNA, ${ }^{-1}$ Luigi AZARIO, ${ }^{1,2}$ (D) Gian Carlo MATTIUCCI, 1,2 (D) Patrizia CORNACCHIONE, \\ (1) Valentina LANCELLOTTA, (1) Maria ANTONIETTA GAMBACORTA, ${ }^{1,2}$ (D) Vincenzo VALENTINI ${ }^{1,2}$
}

\author{
1Fondazione Policlinico Universitario A. Gemelli IRCCS, Rome-Italy \\ ${ }^{2}$ Università Cattolica del Sacro Cuore, Rome-Italy
}

\begin{abstract}
SUMMARY
Salvage radiotherapy is a valid option for locally recurrent prostate cancer (PC) patients. Two of the treatment options are as follows: interventional radiotherapy or stereotactic body radiotherapy (SBRT). SBRT has the main advantage as a noninvasive procedure. Synthetic gel injection into the prostate and rectum space, as a novel technique in prostate radiotherapy, spares the anterior rectal wall from a high dose. Inflammatory bowel disease, including Crohn's disease and ulcerative colitis (UC), contraindicate external beam radiotherapy due to increased bowel complication risk. In this study, we describe the first PC patient with UC in remission treated with salvage SBRT and hydrogel rectal spacer. After ten months of follow-up, no bowel symptoms were reported, and SBRT provided both biochemical and clinical response. Even patients with UC and radio-recurrent PC may be viable salvage SBRT candidates.

Keywords: Hydrogel rectal spacer; recurrent prostate cancer; re-irradiation; stereotactic radiotherapy.

Copyright $\odot$ 2020, Turkish Society for Radiation Oncology
\end{abstract}

\section{Introduction}

Recurrence after primary PC treatment commonly occurs in the prostate gland.[1] Patients with local recurrence, absent metastatic disease, and sufficient life expectancy can be considered for local salvage, such as prostatectomy (RP), cryotherapy (SCT), high-intensity focused ultrasound and re-irradiation. Salvage treatment increases bladder and rectal injury risk and demonstrates augmented toxicity compared with primary treatment, even if toxicity profiles differ.[2]

Re-irradiation can be performed with interventional radiotherapy like brachytherapy (BT), and more recently, with stereotactic body radiation therapy (SBRT). High-dose per fraction radiobiology and tis- sue sparing abilities encourage BT/SBRT use. SBRT, compared with $\mathrm{BT}$, has the main advantage of being a noninvasive procedure.

Many retrospective studies suggest prostate-specific antigen (PSA) control with a low severe rectal and bladder injury incidence in selected patients without pre-existing toxicity.[3-5] Synthetic gel injection between the prostate and rectum, as a novel technique, protects the anterior rectal wall from high-radiationdoses, providing a novel approach to minimize rectum radiation in PC patients with increased late toxicity risk. [6] Inflammatory bowel disease, including Crohn's disease and ulcerative colitis (UC), contraindicates RT because of increased gastrointestinal toxicity risk and subsequent disease exacerbation.[7]
Received: July 02, 2019

Accepted: August 05, 2019

Online: January 08, 2020

Accessible online at:

www.onkder.org

OPEN ACCESS This work is licensed under a Creative Commons

Attribution-NonCommercial 4.0 International License.
Antonio PIRAS, MD

Department of Diagnostic Imaging,

A. Gemelli University Polyclinic Foundation IRCCS,

UOC of Oncological Radiotherapy,

Oncological Radiotherapy and Hematology,

Rome-Italy

E-mail: antoniopiras88@gmail.com 
In this study, we describe a PC patient with UC treated with salvage SBRT in combination with hydrogel rectal spacer.

\section{Case Report}

In January 2018, during a follow-up/control visit, a 66-year-old patient was diagnosed with locally recurrent PC, instrumental-detected at our hospital, showing PSA relapse values of $9.32 \mathrm{ng} / \mathrm{ml}$, PSA velocity of $2.1 \mathrm{ng} / \mathrm{mL} / \mathrm{month}$. The patient was treated fifteen years ago with combined radio-hormone therapy as primary treatment (initially, prostate adenocarcinoma, Gleason score of $3+3=6$, PSA value of $4.19 \mathrm{ng} / \mathrm{ml}$, cT3aN0M0) in our institution. Particularly, the primary treatment consisted of neoadjuvant and adjuvant androgen deprivation therapy combined with three-dimensional conformal radiotherapy to the prostate (total dose 73.8 Gy) and the seminal vesicles (total dose $55.8 \mathrm{~Gy}$ ) with standard (1.8 Gy) fractionation.

A multiparametric magnetic resonance imaging (MRI) of the prostate revealed PC recurrence with a focal lesion within the peripheral right prostatic lobe portion, without extra-prostatic extension measuring $18 \mathrm{~mm}$ at the largest point (Fig.1a). A whole body PET CT with 68Ga-PSMA revealed increased metabolic activity in the right posterior side of the bladder neck, without any other suspicious regional and distant findings (Fig. 1b).

Patient's comorbidities included UC, benign prostatic hyperplasia, treated with transurethral resection three years before, and asymptomatic ischemic heart disease, treated with percutaneous coronary intervention (PCI) in 2011.

UC was diagnosed in 1982, with a disease course characterized by very long remission periods, with only few exacerbations requiring medical management for about 30 years. The last exacerbation was successfully treated with steroid therapy in June 2017. The patient was in a remission phase after primary irradiation. A colonoscopy, in January 2018, showed no signs of inflammation and the patient did not complaint any symptoms of active UC.

The patient decided after counseling against BT but for focal stereotactic body radiotherapy (SBRT) with hydrogel rectal spacer. SBRT was planned as monotherapy due to previous irradiation and UC.

\section{Hydrogel Rectal Spacer Injection, and SBRT Plan- ning and Delivery}

At the Interventional Oncology Center of the Gemelli ART (Advanced Radiation Therapy) [8], 10 cc of a synthetic hydrogel, SpaceOAR (Augmenix Inc., Waltham, MA, USA) were transperineally injected in the mid gland fat plane between Denonvilliers' fascia and the anterior rectal wall, with an ultrasound stepper and transrectal ultrasound (TRUS) in sterile dorsal lithotomy position following the procedure as described by Montoya et al.[9] No complication occurred during the injection. CT simulation scan and MR simulation scan using ViewRay (MRIdian MRI-Guided Linac) confirmed the correct gel position (Fig. 2).
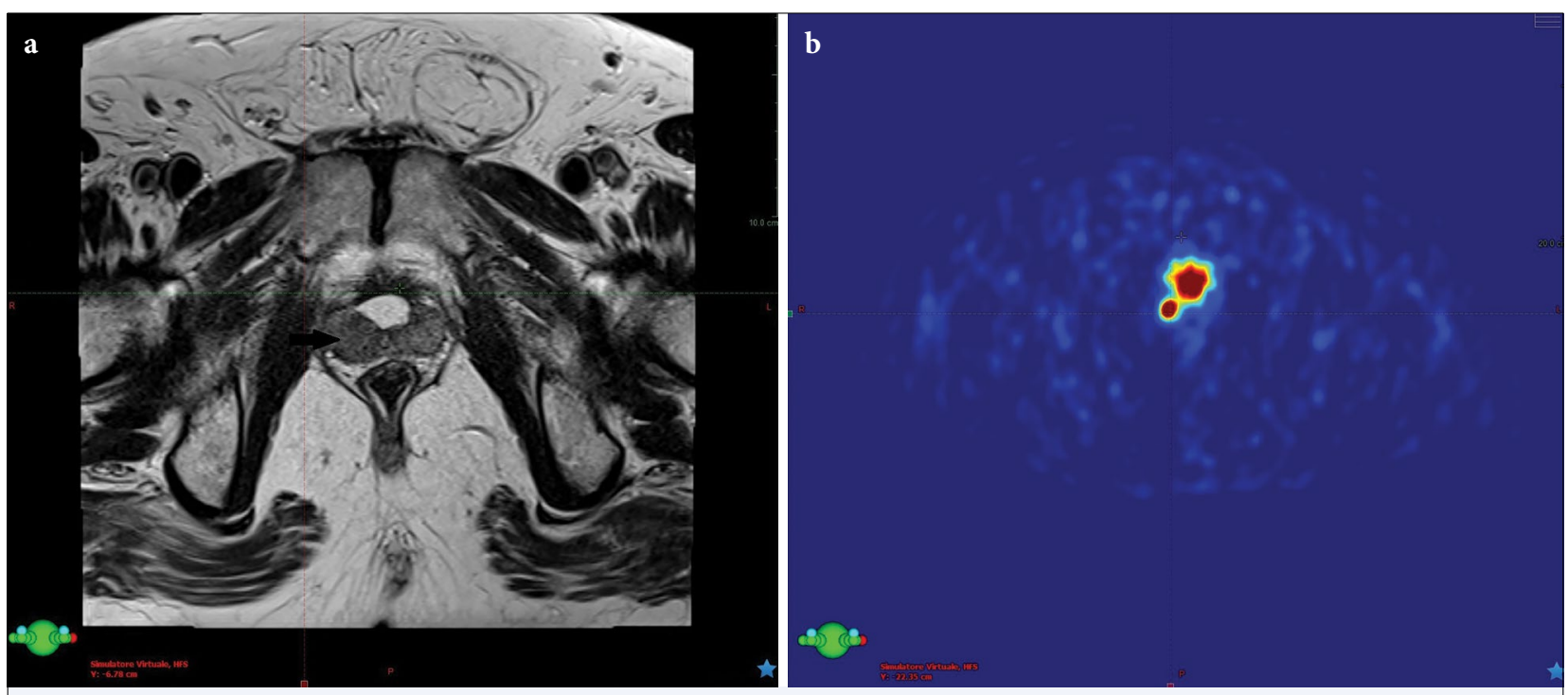

Fig. 1. Axial MR images showing a focal lesion within the peripheral portion of the right prostatic lobe that is hypointense in the T2-weighted sequence (Fig. 1a); PET CT with 68Ga-PSMA images showing increased metabolic activity in the same area (Fig. 1b). 
Fifteen days later, the patient underwent a noncontrast-enhanced multi-slice CT scan (GE MultisliceLightSpeed $^{\text {ma }} 16$ helical CT scanner, GE Medical Systems, Waukesha, WI) with $1.25 \mathrm{~mm}$ slice thickness. Scans were acquired in a supine position, with the Kneefix 3 immobilization device (CIVCO Radiotherapy). The patient received microlax enema before the CT simulation appointment, emptied his bladder and drank $450 \mathrm{ml}$ of water $30 \mathrm{~min}$ before scanning before the intervention. Prostate index tumor was defined by MRI and PET and was considered as the gross tumor volume (GTV). The GTV was delineated using image fusion of MRI and ${ }^{68} \mathrm{Ga}$-PSMA PET CT with planning CT. The clinical target volume (CTV) was defined as the GTV plus $2 \mathrm{~mm}$. The planning target volume (PTV) included the CTV plus 3-mm margin. Rectum (from the anus to the rectosigmoid junction), bladder (including wall and lumen) and left and right femoral heads were defined as organs at risk (OARs), in accordance with the SABR Consortium UK guidelines.[10]

Figure 3 shows a CT simulation image indicating the hydrogel rectal spacer distribution, the target and OARs.

Treatment planning was performed in Eclipse External Beam Planning system V11.0.31 (Varian Medical Systems, Palo Alto, CA) using volumetric-modulated arc therapy (VMAT) (RapidArc ${ }^{\mathrm{Tw}}$ ).

The plan was optimized using the inverse planning Progressive Resolution Optimizer (PRO3), and the fi- nal dose calculation was performed using Anisotropic Analytical Algorithm AAA 11.0.31 with a calculation grid size of $1.25 \mathrm{~mm}$.

Five fractions of $5 \mathrm{~Gy}$ for a total dose of $25 \mathrm{~Gy}$ was prescribed to the $80 \%$ isodose line. Intended planning criteria was V95\% >99\% for the CTV and V95\% >95\% for PTV. The dose distribution to the OAR was optimized taking into account previous treatment doses keeping as low as reasonably achievable the volume of rectum and bladder receiving a cumulative dose of more than $100 \mathrm{~Gy}_{3}$ and $110 \mathrm{~Gy}_{3}$, respectively.[11]

Figure 4 shows the dose distribution and the dosevolume histogram of the SBRT plan.

The treatment was delivered using Edge ${ }^{\bullet}$ Radiosurgery System (Varian Medical Systems, Palo Alto, CA) on alternating days. Daily target localization was achieved through cone-beam computed tomography and six-degree of freedom set-up correction.

The treatment was completed without interruptions. The patient only complaint mild dysuria two weeks after SBRT. After ten months, no urinary or bowel symptoms were reported, the PSA value was $1.31 \mathrm{ng} / \mathrm{ml}$, and an MRI showed a slight reduction of the lesion ( $15 \mathrm{~mm}$ maximum diameter) without abnormalities on high b-value DWI.

The patient signed an informed consent describing in detail the chosen therapeutic approach and gave consent for the use of data for this research.
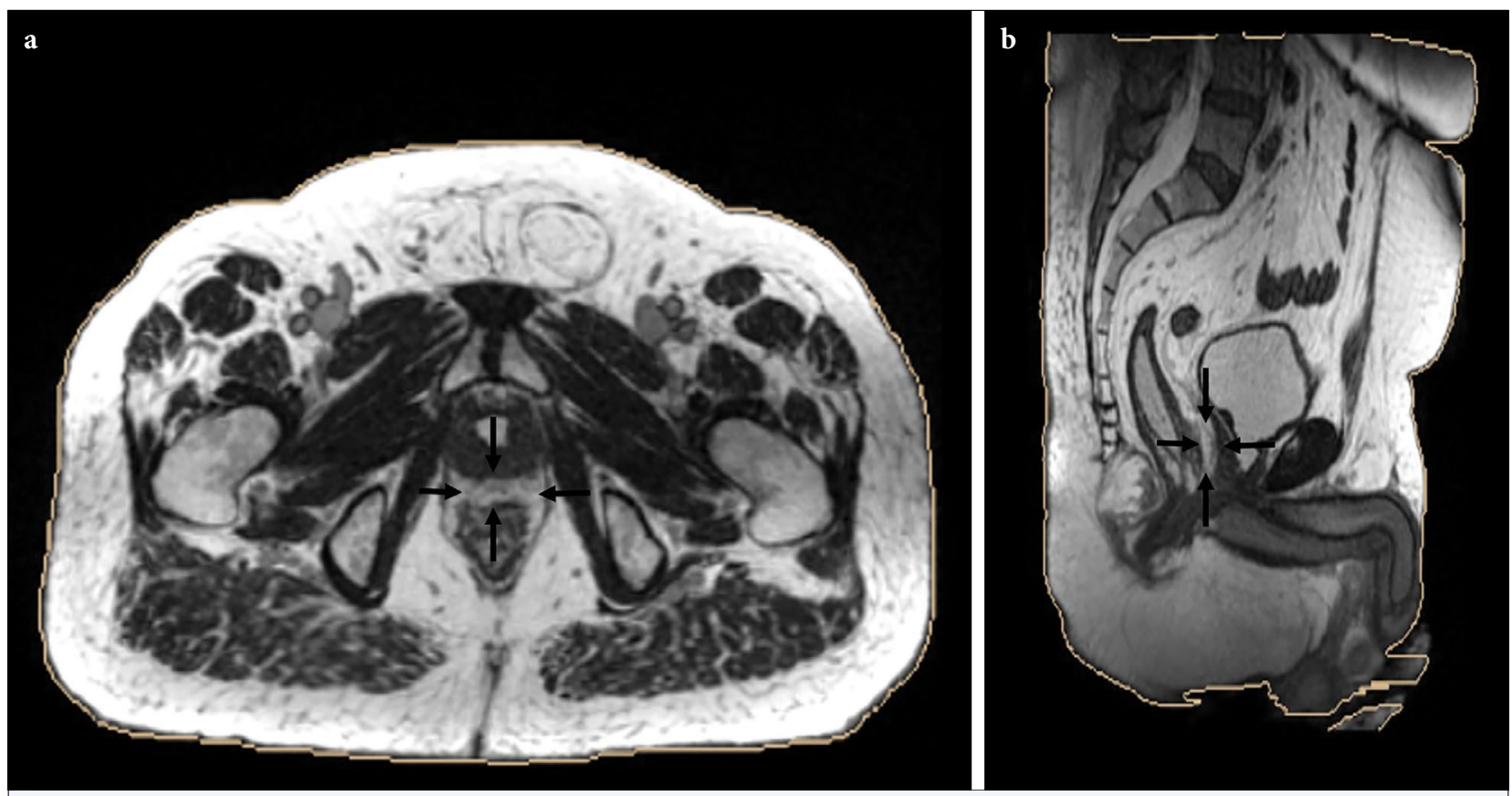

Fig. 2. Axial (Fig. 2a) and sagittal (Fig. 2b) MR simulation imaging showing the position of the spacer gel (black arrows) between the rectum and the prostate. 

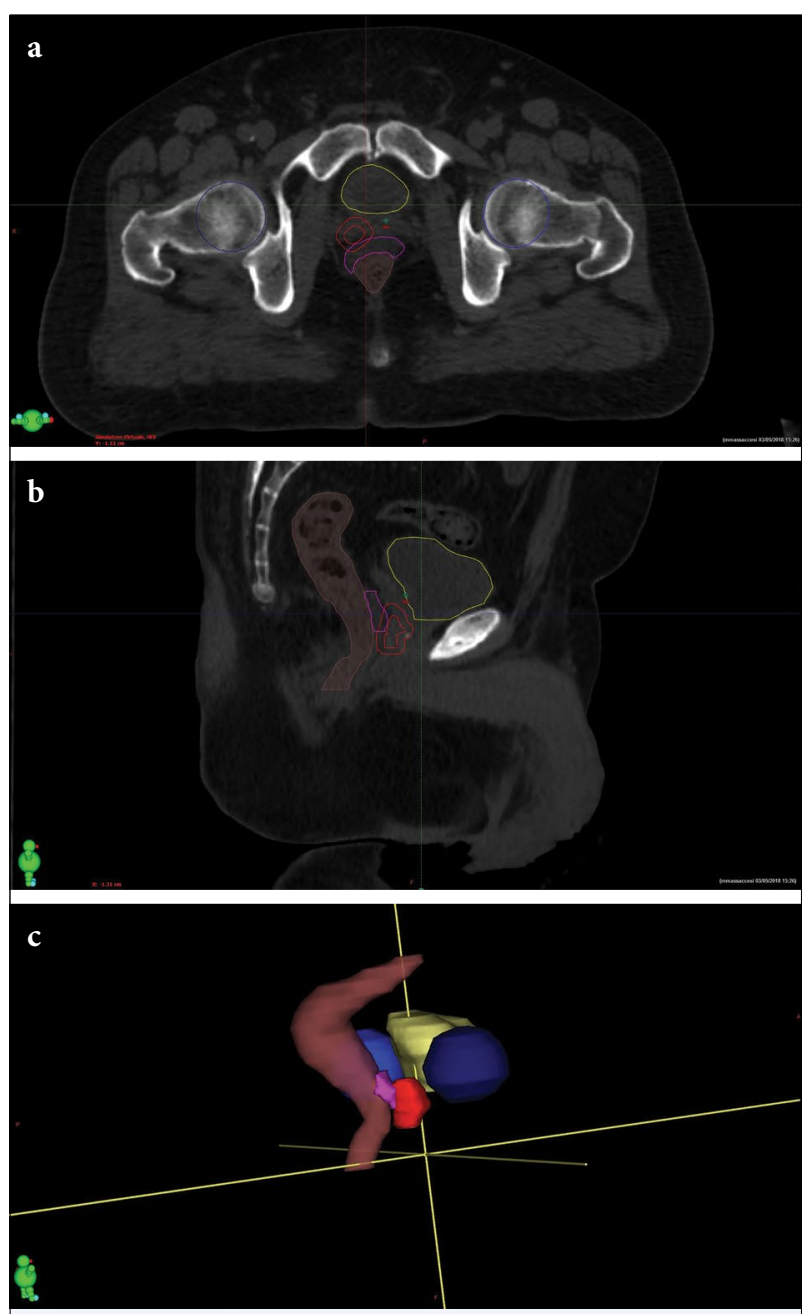

Fig. 3. Axial (Fig. 3a), sagittal (Fig. 3b) CT simulation images, and three-dimensional reconstruction (Fig. 3c) showing the hydrogel spacer distribution (purple contour), the GTV (inner red contour), the PTV (outer red contour), the bladder (yellow contour), the rectum (brown contour), and the femoral heads (blue contour).

\section{Discussion}

We used a hydrogel rectum spacer to perform salvage SBRT in a patient with radio-recurrent prostate cancer and UC. Our patient was not on pharmacologic therapy and, as such, had a favorable prognosis with regards to toxicity stemming from RT.[12] Several studies report the feasibility of hydrogel rectal spacers in patients receiving primary radiation for prostate cancer.[13-18] A recent UC patient case report demonstrated the successful treatment with Ir-192 brachytherapy for primary PC, after SpaceOAR gel injection. The patient reported no bowel issues following HDR brachytherapy after one day, one week and five months, respectively.[19]
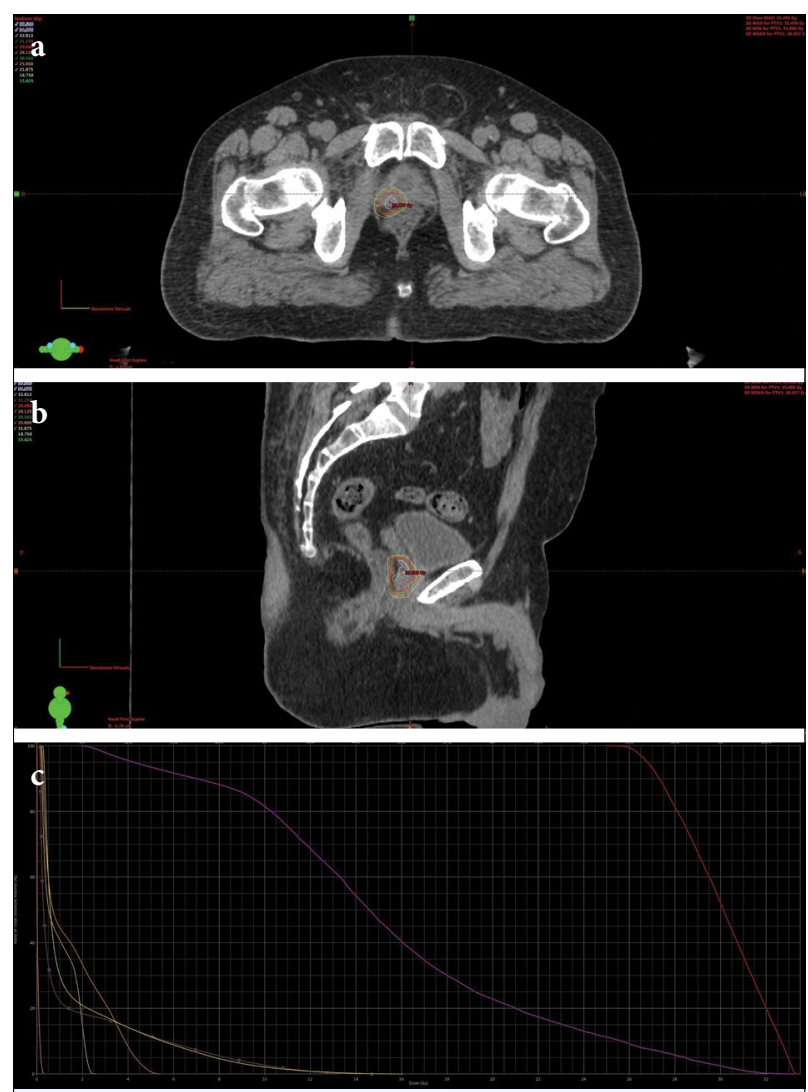

Fig. 4. Axial (Fig. 4a), sagittal (Fig. 4b) CT simulation images, and dose-volume histogram (Fig. 3c) of the SBRT plan.

Studies seldom investigate rectal spacer placement in previously irradiated prostates. Injection feasibility remains unclear in externally irradiated patients (e.g. brachytherapy). A multicentre phase II study, with concluded inclusion, but pending results, evaluating the effectiveness of hyaluronic acid gel injection after low-dose whole-gland prostate brachytherapy with iodine seeds in hyper-selected patients with a high probability of isolated local recurrence could deliver more insights.

Temporary spacer injection, with absorbable polyethylene glycol rectal hydrogel to reduce rectal dose before prostate radiation decreased maximum and mean rectal doses in a man with prior pelvic radiotherapy. [20] One year later, the same group reported initial experience using salvage ${ }^{125} \mathrm{I}$ brachytherapy with hydrogel rectal spacer in 11 prostate cancer patients. Spacing was achieved, with increased median space between the prostate and rectum, in 8/11 (73\%) patients but was not possible in three patients with fibrosis and adhesions. One patient developed a prostate-rectal fistula.[21] 


\section{Conclusion}

UC is commonly a relative contraindication for definitive RT in PC patients, and even more discussible in case of re-irradiation. Our patient case demonstrated the successful focal salvage SBRT use with a hydrogel rectal spacer to limit radiation-induced bowel toxicity. No late gastrointestinal toxicities have been reported after ten months follow-up following spacer placement and subsequent focal SBRT. These results, in combination with previous studies, demonstrate that even patients with UC and radio-recurrent PC might be viable candidates for salvage SBRT in combination with hydrogel rectal spacer limiting rectal toxicity.

Acknowledgments: Authors thank doctor Franziska Michaela Lohmeyer for language editing.

Informed consent: Written informed consent was obtained from the patient for the publication of the case report and the accompanying images.

Peer-review: Externally peer-reviewed.

Conflict of Interest: No conflict of interest to declare.

Financial Disclosure: No funding was received.

Authorship contributions: Concept - M.M.; Design M.M., L.C., L.T., A.P.; Supervision - M.M., G.C.M., V.V., L.A., M.A.G.; Materials - M.M., V.L., P.C., A.P.; Data collection \&/or processing - M.M., L.C., A.P., S.M., L.T.; Analysis and/or interpretation - M.M., A.P.; Literature search - M.M., L.T.; Writing - M.M., L.T., A.P., L.C., S.M.; Critical review G.C.M., L.A., V.V., M.A.G.

\section{References}

1. Agarwal PK, Sadetsky N, Konety BR, Resnick MI, Carroll PR; Cancer of the Prostate Strategic Urological Research Endeavor (CaPSURE). Treatment failure after primary and salvage therapy for prostate cancer: likelihood, patterns of care, and outcomes. Cancer 2008;112(2):307-14.

2. Haj-Hamed M, Karivedu V, Sidana A. Salvage treatment for radio-recurrent prostate cancer: a review of literature with focus on recent advancements in image-guided focal salvage therapies. Int Urol Nephrol 2019;51(7):1101-6.

3. Leroy T, Lacornerie T, Bogart E, Nickers P, Lartigau E, Pasquier D. Salvage robotic SBRT for local prostate cancer recurrence after radiotherapy: preliminary results of the Oscar Lambret Center. Radiat Oncol 2017;12(1):95.

4. Jereczek-Fossa BA, Beltramo G, Fariselli L, Fodor C, Santoro L, Vavassori A, Zerini D, et al. Robotic image- guided stereotactic radiotherapy, for isolated recurrent primary, lymph node or metastatic prostate cancer. Int J Radiat Oncol Biol Phys 2012;82(2):889-97.

5. Fuller DB, Wurzer J, Shirazi R, Bridge SS, Law J, Mardirossian G. High-dose-rate stereotactic body radiation therapy for postradiation therapy locally recurrent prostatic carcinoma: Preliminary prostate-specific antigen response, disease-free survival, and toxicity assessment. Pract Radiat Oncol 2015;5(6):e615-23.

6. Fiorentino A, Giaj Levra N, Mazzola R, Fersino S, Ricchetti F, Ballario R, et al. Stereotactic body radiation therapy with hydrogel spacer: a salvage reirradiation strategy for prostate cancer recurrence. Minerva Urol Nefrol 2016;68(5):464-6.

7. Tromp D, Christie DR. Acute and late bowel toxicity in radiotherapy patients with inflammatory bowel disease: a systematic review. Clin Oncol(R Coll Radiol) 2015;27(9):536-41.

8. Kovács G, Tagliaferri L, Valentini V. Is an Interventional Oncology Center an advantage in the service of cancer patients or in the education? The Gemelli Hospital and INTERACTS experience. J Contemp Brachytherapy 2017;9(6):497-8.

9. Montoya J, Gross E, Karsh L. How I Do It: Hydrogel spacer placement in men scheduled to undergo prostate radiotherapy. Can J Urol 2018;25(2):9288-93.

10.SABR UK Consortium. Stereotactic ablative body radiation therapy (SABR): a resource. 2016. Available from: https://www.sabr.org.uk/wp-content/uploads/2019/04/SABRconsortium-guidelines-2019v6.1.0.pdf

11. Abusaris H, Storchi PR, Brandwijk RP, Nuyttens JJ. Second re-irradiation:efficacy, dose and toxicity in patients who received three courses of radiotherapy with overlapping fields. Radiother Oncol 2011;99(2):235-9.

12. Murphy CT, Heller S, Ruth K, Buyyounouski MK, Weinberg D, Uzzo RG, et al. Evaluating toxicity from definitive radiation therapy for prostate cancer in men with inflammatory bowel disease: Patient selection and dosimetric parameters with modern treatment techniques. Pract Radiat Oncol 2015;5(3):e215-22.

13. Ruciński A, Bauer J, Campbell P, Brons S, Unholtz D, Habl G, et al. Preclinical investigations towards the first spacer gel application in prostate cancer treatment during particle therapy at HIT. Radiat Oncol 2013;8:134.

14. Mariados N, Sylvester J, Shah D, Karsh L, Hudes R, Beyer D, et al. Hydrogel Spacer Prospective Multicenter Randomized Controlled Pivotal Trial: Dosimetric and Clinical Effects of Perirectal Spacer Application in Men Undergoing Prostate Image Guided Intensity 
Modulated Radiation Therapy.Int J Radiat Oncol Biol Phys 2015;92(5):971-77.

15. Hamstra DA, Mariados N, Sylvester J, Shah D, Karsh L, Hudes R, et al. Continued Benefit to Rectal Separation for Prostate Radiation Therapy: Final Results of a Phase III Trial. Int J Radiat Oncol Biol Phys 2017;97(5):976-85.

16. Karsh LI, Gross ET, Pieczonka CM, Aliotta PJ, Skomra CJ, Ponsky LE, et al. Absorbable Hydrogel Spacer Use in Prostate Radiotherapy: A Comprehensive Review of Phase 3 Clinical Trial Published Data. Urology 2018;115:39-44.

17. van Gysen K, Kneebone A, Alfieri F, Guo L, Eade T. Feasibility of and rectal dosimetry improvement with the use of SpaceOAR ${ }^{\circledast}$ hydrogel for dose-escalated prostate cancer radiotherapy. J Med Imaging Radiat Oncol 2014;58(4):511-6.

18. Hedrick SG, Fagundes M, Case S, Renegar J, Blakey M, Artz M, et al. Validation of rectal sparing throughout the course of proton therapy treatment in prostate cancer patients treated with SpaceOAR ${ }^{\circledR}$.J Appl Clin Med Phys 2017;18(1):82-9.

19. Trager M, Greenberger B, Harrison AS, Keller J, Den RB. SpaceOAR to improve dosimetric outcomes for monotherapy high-dose-rate prostate implantation in a patient with ulcerative colitis. J Contemp Brachytherapy 2018;10(6):577-82.

20. Nguyen PL, Devlin PM, Beard CJ, Orio PF 3rd, O'Leary MP, Wolfsberger LD, et al. High-dose-rate brachytherapy for prostate cancer in a previously radiated patient with polyethylene glycol hydrogel spacing to reduce rectal dose: case report and review of the literature. Brachytherapy 2013;12(1):77-83.

21. Mahal BA, Ziehr DR, Hyatt AS, Neubauer-Sugar EH, O'Farrell DA, O'Leary MP, et al. Use of a rectal spacer with low-dose-rate brachytherapy for treatment of prostate cancer in previously irradiated patients: Initial experience and short-term results. Brachytherapy 2014;13(5):442-9. 